\title{
Development of a Method Combining Peptidiscs and Proteomics to Identify, Stabilize and Purify a Detergent-Sensitive Membrane Protein Assembly
}

John William Young ${ }^{1,4,}$ Irvinder Singh Wason ${ }^{1,4}$, Zhiyu Zhao ${ }^{1}$, Sunyoung Kim ${ }^{2}$, Hiroyuki Aoki $^{2}$, Sadhna Phanse ${ }^{2}$, David G Rattray ${ }^{3}$, Leonard J. Foster ${ }^{3}$, Mohan Babu ${ }^{2}$ and Franck Duong van $\mathrm{Hoa}^{1, *}$

${ }^{1}$ Department of Biochemistry and Molecular Biology, Faculty of Medicine, Life Sciences Institute, University of British Columbia, Vancouver, British Columbia, Canada. ${ }^{2}$ Department of Biochemistry, Faculty of Science, University of Regina, Canada. ${ }^{3}$ Department of Biochemistry and Molecular Biology, Faculty of Medicine, Michael Smith Laboratory, University of British Columbia ${ }^{4}$ These authors contributed equally to this work.

* To whom correspondence should be addressed: Fduong@mail.ubc.ca

Keywords: Peptidisc, Membrane Proteome, Membrane Protein Purification, Affinity Purification/Mass Spectrometry (AP/MS), Sec translocon.

\section{ABSTRACT}

The peptidisc membrane mimetic enables global reconstitution of the bacterial membrane proteome into water-soluble detergent-free particles, termed peptidisc libraries. We present here a method that combines peptidisc libraries and chromosomal-level gene tagging technology with affinity purification and mass spectrometry (AP/MS) to stabilize and identify fragile membrane protein complexes that exist at native expression levels. This method circumvents common artifacts caused by bait protein overproduction and protein complex dissociation due to lengthy exposure to detergents during protein isolation. Using the $E$. coli Sec system as a case study, we identify an expanded version of the translocon, termed the HMD complex, consisting of 9 different integral membrane subunits. This complex is stable in peptidiscs but dissociates in detergent. Guided by this native-level proteomic information, we design and validate a procedure that enables purification of the HMD complex with minimal protein dissociation. These results highlight the utility of peptidiscs and AP/MS to discover and stabilize fragile membrane protein assemblies. 


\section{INTRODUCTION}

Early efforts to characterize membrane protein interaction networks - or the "membrane protein interactome" - have mostly relied on the over-production of bait proteins followed by membrane solubilization with detergents (1-5). After isolation of the bait, potential interactors are identified by mass spectrometry or immunoblotting (1-3, 5). Although straightforward, there are major drawbacks to this method. First, detergent micelles, which are necessary to maintain membrane protein solubility, tend to dissociate fragile multisubunit complexes, causing protein-protein associations to be lost (1-4,6-8). Detergents must also be carefully removed before mass spectrometry analysis, which tends to decrease the efficiency of protein identification. Second, plasmid-based over-expression of the bait protein often perturbs cell envelope biogenesis and potentially alters the stoichiometry and specificity of protein interactions, leading to adverse effects on cell physiology and complex identification $(4,9,10)$.

Several chromosomal-level tagging approaches have been developed recently to bypass the need for bait protein overproduction $(1,11,12)$. Work from our group and others has shown the utility of chromosomal tagging approaches to map protein interaction networks in bacteria, yeast, and mammalian cells $(1,2,11-13)$. To circumvent the adverse effects of detergents, several membrane mimetics have been developed to isolate membrane proteins in a water-soluble state $(4,14-16)$. Results from our group have shown that the peptidisc enables reconstitution of a significant fraction of the membrane proteome in a form suitable to mass spectrometry analysis (Figure 1) $(4,16)$.

In this report, we combine these two recent developments to identify and stabilize membrane protein interactions at native expression levels, and we use this information to guide the development of a protocol to purify a membrane protein complex of interest with minimal interference from detergent. We start with an E. coli strain that is modified at the chromosomal-level with a sequential peptide affinity (SPA) tag inserted at a specific gene position, thereby creating a C-terminal fusion with the protein of interest. $(1,10)$. After reconstitution of the membrane proteome into peptidiscs, the bait and its co-purifying interactors are identified by AP/MS analysis. To solidify our initial findings, and to better characterize the interactome of our protein of interest, we then repeat this peptidisc-AP/MS 
workflow using SPA-tagged strains for the most prominent interactors identified in our initial experiment. To differentiate between enriched interactors and non-specific protein contaminants, we performed this workflow in parallel using an E. coli strain with no SPA tag.

As a case study, we apply this Peptidisc-AP/MS workflow toward characterizing the membrane interactome of the highly conserved bacterial translocon - also known as the SecYEG complex - which forms a protein-conducting channel across the inner membrane in gram-negative bacteria $(17,18)$. We selected the translocon as an experimental testbed because it is known to associate dynamically with a wide variety of integral membrane protein subunits. These include the membrane-integrated SecDFyajC complex and the membrane insertase YidC, as well as the membrane-tethered periplasmic chaperones YfgM and PpiD (3, 4, 19-23). Previous biochemical studies have isolated a multi-subunit complex termed the bacterial "holo-translocon" (HTL) - consisting of SecYEG, SecDFyajC, and YidC (22, 24-26). However, the HTL purification is technically challenging, requiring multiple different tags and chromatography steps, as well as additional specific phospholipids to minimize the dissociating effects of detergent $(22,24,27)$.

Using Peptidisc-AP/MS workflow described above, we identify an expanded version of the holo-translocon in the native membrane, which we term the "HMD" complex, consisting of SecYEG, the SecDFyajC, and YidC subcomplexes, plus the periplasmic chaperones YfgM and PpiD. Having shown that this assembly can be isolated in peptidiscs, we use this information to develop a protocol that enables overproduction and biochemical purification with minimal protein dissociation.

\section{MATERIALS AND METHODS}

\section{Reagents}

Tryptone, yeast extract, $\mathrm{Na}_{2} \mathrm{HPO}_{4}, \mathrm{KH}_{2} \mathrm{PO}_{4}, \mathrm{NaCl}$, and Tris-base were obtained from Bioshop Canada. Ampicillin, kanamycin, and arabinose were purchased from GoldBio. n-dodecyl- $\beta$ d-maltoside (DDM) was purchased from Anatrace. ANTI-FLAG M2 affinity gel was purchased from Sigma. Peptidiscs (purity $>80 \%$ ) were obtained from Peptidisc Biotech Canada. All other chemicals were obtained from Fisher Scientific Canada. 


\section{Plasmids and strains}

E. coli SPA (Sequential Peptide Affinity)-tagged strains were from the Babu laboratory collection (1). The strains used in this study were as follows: DY330 (non-tagged parental control strain), b3300 (SecY-SPA), b0408 (SecD-SPA), b0441 (PpiD-SPA), and b2513 (YfgM-SPA). Plasmids pBad22-hisEYG, pBad33-DFyajC, pBad22-hisYidC, and pBad33$Y f g M_{H i s}-P p i D$ have been previously described (4, 5, 28-30). All plasmids generated in this study were cloned using the PIPE method (31). The genes for SecDF were amplified from pBad33-DFyajC and inserted into pBad22-hisEYG, generating the plasmid pBad22hisEYGDF. To express the bacterial holo-translocon (HTL; a complex of SecYEG with SecDFyajC and YidC (22)), the gene for YidC without its affinity tag was amplified from pBad22-hisYidC and inserted into pBad22-hisEYGDF to form the plasmid pBad22-HTL. The His-tag was deleted from pBad33-YfgM $M_{H i s}-P p i D$ to generate $\mathrm{pBad} 33-Y f g M-P p i D$. All constructs were verified by DNA sequencing (Genewiz).

\section{Growth of SPA-tagged strains and membrane preparation}

All strains except the DY330 control strain were revived on Luria Bertani (LB)-agar plates supplemented with $25 \mu \mathrm{g} / \mathrm{mL}$ kanamycin. Strain DY330 was revived on a LB-agar plate without antibiotics. For each strain, a single colony was used to inoculate a $10 \mathrm{~mL}$ overnight culture in LB media (plus antibiotic, where appropriate). Overnight cultures were grown overnight at $37^{\circ} \mathrm{C}$ with shaking. The following day, overnight cultures were diluted $1 / 100$ into $1 \mathrm{~L}$ fresh LB media and grown for a further 6 hours until $\mathrm{OD}_{600} \sim 1$. Cells were harvested by centrifugation $(6,000 \mathrm{x} \mathrm{g}, 10$ minutes $)$ and resuspended in TSG (50 mM Tris $\mathrm{HCl} \mathrm{pH} 8 ; 50$ $\mathrm{mM} \mathrm{NaCl} ; 10 \%$ glycerol) buffer containing $1 \mathrm{mM}$ PMSF before being Dounce homogenized and lysed on a Microfluidizer (12,000 psi, three passes). Lysates were centrifuged at 6,000 x $\mathrm{g}$ for 10 minutes to remove unbroken cells. The membrane fraction was collected by ultracentrifugation $(100,000 \mathrm{x}$ g, 30 minutes) in a Ti70 rotor. After centrifugation, the supernatant was discarded, and the membrane pellet was resuspended in TSG buffer.

\section{Preparation of peptidisc libraries}

Membranes ( 1-2 mg) were solubilized with $0.5 \%$ DDM in $1 \mathrm{~mL}$ volume on ice for 15 minutes. Insoluble material was removed by ultracentrifugation (100,000 x $g, 15$ minutes). The detergent-solubilized material was immediately mixed with a 4:1 excess of $\mathrm{NSP}_{R}$ peptidisc peptide in a $15 \mathrm{~mL} 100 \mathrm{kDa}$ cutoff Amicon concentrator. To form a peptidisc library, the solubilized membrane-peptide mixture was diluted to $10 \mathrm{~mL}$ in TSG buffer. The 
mixture was concentrated to $\sim 1 \mathrm{~mL}$ by centrifugation $(3,000 \mathrm{x} \mathrm{g}, 10$ minutes $)$ at $4^{\circ} \mathrm{C}$ before being diluted back to $10 \mathrm{~mL}$ and re-concentrated to $\sim 1 \mathrm{~mL}$.

\section{FLAG pulldowns in peptidisc}

About $1 \mathrm{mg}$ peptidisc libraries were loaded onto $50 \mu \mathrm{L}$ ANTI-FLAG M2 affinity gel and incubated at $4^{\circ} \mathrm{C}$ overnight with gentle shaking. The following day, the flow-through was collected, and the resin was washed with $1 \mathrm{~mL}$ TSG buffer. Five additional $1 \mathrm{~mL}$ washes were performed to minimize non-specific binding to the resin. Bound proteins were eluted in $100 \mu \mathrm{L} 100 \mathrm{mM}$ Glycine $\mathrm{HCl} \mathrm{pH} 3.5$. The sample $\mathrm{pH}$ was adjusted by adding $10 \mu \mathrm{L} 1 \mathrm{M}$ Tris $\mathrm{HCl} \mathrm{pH}$ 8.0. Samples were then digested and STAGE tipped before being analyzed by LCMS/MS exactly as previously described (1).

\section{FLAG pulldowns in detergent}

Membranes were solubilized as described above. About $1 \mathrm{mg}$ of detergent-solubilized material was loaded onto $50 \mu \mathrm{L}$ ANTI-FLAG M2 affinity gel and incubated at $4^{\circ} \mathrm{C}$ overnight with gentle shaking. The next morning, the flow-through was collected, and the resin was washed with $1 \mathrm{~mL}$ TSG buffer $+0.02 \%$ DDM. Five additional $1 \mathrm{~mL}$ washes were performed to minimize non-specific binding to the resin. Bound proteins were eluted in $100 \mu \mathrm{L} 100 \mathrm{mM}$ Glycine $\mathrm{HCl} \mathrm{pH}$ 3.5. Sample $\mathrm{pH}$ was adjusted by adding $10 \mu \mathrm{L} 1 \mathrm{M}$ Tris $\mathrm{HCl} \mathrm{pH}$ 8. The samples were precipitated with ice-cold acetone to remove detergent (overnight incubation, $\left.4^{\circ} \mathrm{C}\right)$. Precipitated proteins were pelleted by centrifugation $(10,000 \mathrm{~g}, 10$ minutes $)$, and the supernatant was removed using a vacuum aspirator. Protein pellets were dried at $42^{\circ} \mathrm{C}$ for 10 minutes. Samples were then digested and STAGE tipped before being analyzed by LCMS/MS exactly as previously described (1).

\section{Analysis of mass spectrometry data}

Analysis was performed using MaxQuant version 1.6.17.0 (32, 33). The search was performed against a database comprised of the protein sequences from the source organism (E. coli $\mathrm{K} 12$ ) plus common contaminants using the following parameters: peptide mass accuracy $<5$ ppm; fragment mass accuracy $0.006 \mathrm{Da}$; trypsin enzyme specificity; fixed modifications, carbamidomethyl; variable modifications, methionine oxidation, deamidated $\mathrm{N}, \mathrm{Q}$, and $\mathrm{N}$-acetyl peptides. Proteins were quantified from 1 peptide identification. Only 
those peptides exceeding the individually calculated $99 \%$ confidence limit (as opposed to the average limit for the whole experiment) were considered as accurately identified.

\section{Capturing labile interactors of the SecYEG complex in peptidiscs}

To over-produce the SecYEG and HTL complexes, plasmids pBad22-EYG and pBad22-HTL were transformed into chemically competent BL21(DE3) cells. To facilitate over-production of the whole "HMD" complex, the plasmid pBad33-YfgM PpiD (containing no affinity tag) was transformed into BL21(DE3) competent cells containing the plasmid pBad22-HTL. Protein expression and membrane isolation were performed as previously described $(28,34$, 35). Briefly, protein expression was induced for $3 \mathrm{hr}$ at $37^{\circ} \mathrm{C}$ after induction with $0.2 \%$ Arabinose at an OD of 0.4-0.7 in 1L LB medium supplemented with appropriate antibiotics. Cell lysis and membrane isolation were as described above. To enrich the inner membrane further, the membrane fraction was layered onto a 2-step 20\%-50\% sucrose gradient in SW41 tubes and re-centrifuged at 200,000 $g$ for 2 hours. Inner Membrane Vesicles (IMVs) were recovered as a distinct brown band near the middle of the gradient and diluted 4-fold in TSG before being pelleted by ultracentrifugation (100,000 x $g$, 15 minutes). To verify successful over-production of all protein subunits, a $5-\mu \mathrm{g}$ aliquot of each membrane preparation was analyzed by $15 \%$ SDS-PAGE followed by Coomassie Blue staining.

For purifications in detergent conditions, IMVs were solubilized with $0.5 \%$ DDM in $1 \mathrm{~mL}$ volume on ice for 15 minutes. Insoluble material was removed by ultracentrifugation $(100,000 \times g, 15$ minutes $)$. A $1 \mathrm{~mL}$ aliquot of the detergent extract was incubated with $50 \mu \mathrm{L}$ Ni-NTA resin for 30 minutes. The resin was washed three times with 10 column volumes (CVs) of TSG buffer $+0.02 \%$ DDM, then eluted in TSG buffer $+0.02 \% \mathrm{DDM}+300 \mathrm{mM}$ Imidazole. Eluted proteins were analyzed by $15 \%$ SDS PAGE and visualized by Coomassie Blue staining. For reconstitution into peptidiscs, a freshly solubilized detergent extract was mixed with a 4:1 excess of peptidisc peptide in a $15 \mathrm{~mL} 100 \mathrm{kDa}$ cutoff Amicon concentrator and reconstituted into peptidisc libraries as previously described (4). The resultant library was injected onto a Superose 6 10/300 column (GE Healthcare) equilibrated in TSG buffer on an AKTA FPLC. Peak fractions were analyzed by SDS-PAGE to verify the presence of all protein subunits. Peak fractions were then pooled and incubated with Ni-NTA resin for 30 minutes at $4^{\circ} \mathrm{C}$. The resin was washed three times with $10 \mathrm{CVs}$ of TSG buffer, then eluted in 
TSG buffer + $300 \mathrm{mM}$ Imidazole. Eluted proteins were analyzed by 15\% SDS PAGE and visualized by Coomassie Blue staining.

\section{RESULTS}

\section{An AP/MS approach to identify the interactome of the Sec translocon}

To benchmark our Peptidisc-AP/MS workflow and identify the SecYEG complex's primary interactors in native membranes, we employed a strain with the sequential peptide affinity (SPA) tag inserted in the $\sec Y$ gene (strain termed SecY-SPA). This strain is perfectly viable, indicating that the SecY protein modified with a C-terminal SPA tag is functional (1). Following cell lysis, the total envelope proteome containing inner and outer membrane proteins was briefly solubilized with n-dodecyl- $\beta$-d-maltoside (DDM). After removing aggregates by ultracentrifugation, a 4:1 mass excess of peptidisc peptide was added, and the detergent micelles were rapidly removed via successive dilution and filtration steps as described in the Materials and Methods. The resulting peptidisc library was then affinity purified over anti-FLAG resin, and the eluate was digested and STAGE tipped before being analyzed in triplicate by LC-MS/MS. A total of 240 proteins were identified in all three replicates (Supplemental File 1). As a graphic representation of these data, the protein list was ranked in alphabetical order, and the intensity value of each protein was plotted as a function of its rank in the list (Figure 2A). To enable identification of the protein contaminants inherent to this type of analysis (e.g., non-specific interactors of the anti-FLAG resin), the peptidisc AP/MS workflow was done in parallel using a library prepared from the parental wild-type strain DY330 (1). In this control, a total of 266 proteins were identified in all three replicates. The protein list was ranked and plotted as described above (Figure 2B).

Comparing the two plots side-by-side, it appears that the SecYEG translocon ancillary membrane subunits PpiD, YfgM, SecD, SecF, SecG, and YidC, as well as SecY itself, are present at far higher intensities in our SecY-SPA pulldown compared to the control. The BamB subunit of the Bam complex is also enriched (red points, Figures 2A and 2B). We note that multiple additional proteins are present at comparable intensities between the SecY-SPA and control pulldowns - including MscK, TufB, SdhA, and multiple ATP synthase subunits (black points, Figures 2A and 2B). Numerous cytosolic ribosomal subunits are also present in 
both pulldowns (grey points, Figure 2A and 2B). The detection of numerous protein contaminants is inherent to AP/MS experiments and proteomic analysis in general $(1,3,4)$.

As an alternative representation of our data, we next plotted the intensities of each protein present in both the SecY-SPA and control pulldowns on the same graph (Figure 2C). Presenting the data in this manner makes it easier to identify specifically enriched proteins versus non-specific contaminants. The result shows that 180 proteins are present in both pulldowns and, consistent with our earlier analysis, the known Sec translocon subunits PpiD, YfgM, SecD, SecF, SecY, SecG, and YidC are clearly enriched (red points). Abundant contaminants such as MscK, TufB, SdhA, and multiple ribosomal subunits are present at roughly equal intensities in both pulldowns.

As a cross-validation assay, we applied our peptidisc AP/MS workflow to three additional strains, encoding for SPA-tagged SecD, PpiD, YfgM. Data were analyzed and visualized as described above (Figure 3A-C). We note that the extent of non-specific protein contamination appears somewhat higher in the SecD and YfgM FLAG pulldown experiments compared to the PpiD FLAG pulldown. However, despite the presence of these protein contaminants, we find these latest results are confirmatory of the findings in our earlier SecY peptidisc AP/MS and suggest a network of interactions between the core Sec translocon, SecD, SecF, YidC, YfgM, and PpiD.

To compare the peptidisc AP/MS workflow against a classical detergent-based AP/MS approach, we performed AP/MS using a detergent extract prepared from the strain SPAtagged for PpiD as described in the Materials and Methods (Figure 4A). PpiD was selected as the bait because its other Sec interactors are well detected in our peptidisc AP/MS experiments above. The experiment was done in parallel using a detergent extract from the parental DY330 strain as a control (Figure 4B). As expected, the results with the PpiD detergent extract show that the protein YfgM is enriched along with PpiD (Figure 4A and 4C) (3). However, many interactors of PpiD identified in our peptidisc AP/MS experiment above - including SecD, SecF, YidC, and SecYEG - are either poorly detected in detergent (i.e., with low intensity) or are undetected. Likely, these interactions are mostly dissociated upon prolonged exposure to detergents, particularly during the washing steps, which are necessary to reduce the abundance of non-specific protein contaminants. We did not observe this protein dissociation in our peptidisc experiments, likely because the reconstitution step to 
stabilize fragile complexes is performed before affinity purification and because washing is performed without detergents.

\section{Biochemical isolation of the HMD complex in peptidiscs}

Altogether, our proteomic data reveals interactions between SecYEG, SecDF, YidC, YfgM, and PpiD in native E. coli membranes. This network of interactions - identified here across multiple peptidisc AP/MS experiments using multiple different baits - likely represents a super-complex containing all subunits of the previously characterized bacterial holotranslocon (SecYEG, SecDF, and YidC), plus the membrane-tethered chaperones YfgM and PpiD. Thus, we are naming this complex the "HMD" (holo-translocon plus Yfgㅆ $\underline{\mathrm{M}}$ and PpiD) complex. Guided by this information, we next designed a protocol to enable its biochemical purification.

We cloned the holo-translocon (HTL) SecYEG-SecDF-YidC subunits into a bacterial expression vector. The protein SecE bears an N-terminal His-tag to facilitate affinity purification of the complex. For over-production of the entire HMD complex, non-tagged YfgM and PpiD were cloned into a second plasmid with different antibiotic resistance.

To verify successful over-production of all protein subunits, we expressed the HTL and HMD complexes in E. coli BL21(DE3) and isolated the inner membranes. In addition, the core translocon SecYEG was expressed side-by-side as a control, and to visualize protein content, the membranes were analyzed by 15\% SDS-PAGE and Coomassie blue staining (Figure 5A). Encouragingly, we observe that all protein subunits of the HMD complex are produced in roughly similar amounts.

Membranes bearing the SecYEG and HMD complexes were solubilized briefly in DDM and affinity-purified in detergent. Aliquots from each purification step were analyzed by SDSPAGE and Coomassie blue staining (Figure 5B). Consistent with our initial proteomic observations, the HMD complex appears largely dissociated, with the PpiD, YfgM, SecDF, and YidC subunits primarily lost in the detergent washing steps. On the other hand, the core translocon SecYEG appears stable during purification in DDM, as expected.

Next, we reconstituted a fresh aliquot of the unpurified HMD detergent extract into peptidiscs. To verify the solubility of our preparation, we first fractionated the sample by size 
exclusion chromatography (SEC) in detergent-free conditions. Visual examination of the SEC chromatogram reveals two peaks - the first corresponds to the reconstituted peptidisc library, while the second corresponds to excess peptidisc peptides (Figure 6A). The peptidisc library clearly elutes after the column's void volume, indicating that the library is watersoluble and free of aggregates. Fractions under the central peak were analyzed by SDS-PAGE (Figure 6B). As expected, all subunits of the HMD complex are present in roughly stoichiometric amounts. These peaks fractions were pooled, and the HMD complex was isolated by Nickel affinity purification. Aliquots from each purification step were analyzed by SDS-PAGE (Figure 6C). Consistent with our earlier proteomic observations, the PpiD, YfgM, SecDF, and YidC subunits co-purify along with SecYEG.

\section{DISCUSSION}

In this study, we develop a method to detect multi-subunit membrane protein complexes which are naturally present in the cell membrane, yet difficult to identify because of their low abundance and facile dissociation when extracted with detergent. Specifically, we combine the peptidisc membrane mimetic with a series of E. coli strains that are modified with a sequential peptide affinity (SPA) tag at the chromosomal-level. This approach maintains expression of the target protein at near-endogenous levels, which minimizes artifacts due to protein overproduction such as misfolding and mis-localization $(1,10)$. This approach also decreases protein complex dissociation because their capture peptidiscs allows to minimize exposure to detergents. After stabilization of the membrane complex in peptidiscs, the SPAtagged bait proteins and their interactors are isolated by affinity chromatography and identified by mass spectrometry. To facilitate identification of background contaminants versus enriched interactors, the pulldown experiment is repeated in parallel using a parental E. coli strain with no SPA tag.

Using the well-characterized bacterial Sec translocon as an experimental model, we perform a series of AP/MS experiments on multiple known Sec ancillary subunits. This work led us to identify an expanded version of the holo-translocon complex consisting of SecYEG, SecDFyajC, YidC, plus the two membrane chaperones YfgM and PpiD. We named it the 


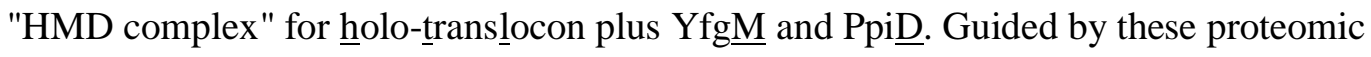
observations, we develop a purification protocol to isolate biochemical amounts of the HMD complex. We start by overproducing the HMD complex with a single affinity tag using $E$. coli expression vectors. We note that the over-produced HMD complex is largely dissociated when attempting purification in detergent conditions. Even mild detergents such as DDM are known to exhibit dissociative and delipidating effects on membrane proteins, particularly during the washing steps that are intrinsic to affinity purification $(4,36,37)$. Therefore, to minimize these detergent effects, we reconstitute the HMD complex into peptidiscs before attempting any purification steps. When analyzed by size-exclusion chromatography (SEC) in detergent-free conditions, we observe that our peptidisc preparation is water-soluble and free of aggregates and that all subunits of the HMD complex co-elute together from the column, indicating that the complex is stabilized in peptidiscs. After pooling the peak fractions from our initial SEC experiment, we can then isolate the HMD complex by affinity purification.

Although this study does not reveal novel protein interactors of the Sec translocon, this work provides the first evidence that the "HMD" complex is an entity present in the native membrane. Other studies have determined that the copy number of SecYEG and interacting partners differs considerably within the cell $(21,38)$. Given these different expression levels, the "HMD" complex identified here is likely to be a highly dynamic assembly in native membranes, rapidly assembling and disassembling into various sub-complexes. This dynamic association would explain the fragility of the complex. Considering this, we do not exclude the possibility that our purified preparation contains a mixture of both complete "HMD" complex and varied sub-complexes. Details regarding the stoichiometry, dynamics, and structure of the HMD complex will need to be presented in future experiments. The identity of any annular lipids that may modulate the HMD complex's activity may also be a promising avenue for future investigation.

This current work represents the results of a pilot study using a series of SPA-tagged E. coli strains. These strains are only a tiny subset of a more extensive strain library available in our laboratory, in which hundreds of $E$. coli membrane protein open-reading frames (ORFs) have been systematically SPA-tagged. A similar public library is also available to characterize the S. cerevisiae membrane proteome $(1,2)$. Other recent work reports the high throughput construction of new strains and cell lines to map protein interaction networks in mitochondria 
and mouse brain tissue using AP/MS $(13,39,40)$. Given the widespread availability of geneediting technology, we anticipate that our Peptidisc-AP/MS approach will be easily expandable to precisely characterize membrane protein interactomes across multiple different organisms and tissue types.

\section{ACKNOWLEDGEMENTS}

This work was supported the Canadian Institutes of Health Research to the FDVH's group. Platform funding for proteomics research in L.J.F.'s group is supported by Genome Canada and Genome BC (264PRO).

\section{REFERENCES}

1. M. Babu et al., Global landscape of cell envelope protein complexes in Escherichia coli. Nat Biotechnol 36, 103-112 (2018).

2. M. Babu et al., Interaction landscape of membrane-protein complexes in Saccharomyces cerevisiae. Nature 489, 585-589 (2012).

3. G. Maddalo et al., Systematic analysis of native membrane protein complexes in Escherichia coli. J Proteome Res 10, 1848-1859 (2011).

4. M. L. Carlson et al., Profiling the E. coli membrane interactome captured in peptidisc libraries. Elife 8, (2019).

5. F. Duong, W. Wickner, Distinct catalytic roles of the SecYE, SecG and SecDFyajC subunits of preprotein translocase holoenzyme. EMBO J 16, 2756-2768 (1997).

6. A. Gokhale, P. Perez-Cornejo, C. Duran, H. C. Hartzell, V. Faundez, A comprehensive strategy to identify stoichiometric membrane protein interactomes. Cell Logist 2, 189-196 (2012).

7. D. S. Chorev et al., Protein assemblies ejected directly from native membranes yield complexes for mass spectrometry. Science 362, 829-834 (2018).

8. G. Angiulli et al., New approach for membrane protein reconstitution into peptidiscs and basis for their adaptability to different proteins. Elife 9, (2020).

9. A. C. Gingras, K. T. Abe, B. Raught, Getting to know the neighborhood: using proximity-dependent biotinylation to characterize protein complexes and map organelles. Curr Opin Chem Biol 48, 44-54 (2019).

10. M. Zeghouf et al., Sequential Peptide Affinity (SPA) system for the identification of mammalian and bacterial protein complexes. J Proteome Res 3, 463-468 (2004).

11. P. Hu et al., Global functional atlas of Escherichia coli encompassing previously uncharacterized proteins. PLoS Biol 7, e96 (2009).

12. M. Babu et al., Sequential peptide affinity purification system for the systematic isolation and identification of protein complexes from Escherichia coli. Methods Mol Biol 564, 373-400 (2009).

13. R. H. Malty et al., A Map of Human Mitochondrial Protein Interactions Linked to Neurodegeneration Reveals New Mechanisms of Redox Homeostasis and NF- $\kappa B$ Signaling. Cell Syst 5, 564-577.e512 (2017).

14. J. Roy, H. Pondenis, T. M. Fan, A. Das, Direct Capture of Functional Proteins from Mammalian Plasma Membranes into Nanodiscs. Biochemistry 54, 6299-6302 (2015). 
15. M. T. Marty, K. C. Wilcox, W. L. Klein, S. G. Sligar, Nanodisc-solubilized membrane protein library reflects the membrane proteome. Anal Bioanal Chem $\mathbf{4 0 5}$, 4009-4016 (2013).

16. J. W. Young et al., His-Tagged Peptidiscs Enable Affinity Purification of the Membrane Proteome for Downstream Mass Spectrometry Analysis. J Proteome Res, (2020).

17. T. A. Rapoport, L. Li, E. Park, Structural and Mechanistic Insights into Protein Translocation. Annu Rev Cell Dev Biol 33, 369-390 (2017).

18. E. Park, T. A. Rapoport, Mechanisms of Sec61/SecY-mediated protein translocation across membranes. Annu Rev Biophys 41, 21-40 (2012).

19. H. Götzke et al., YfgM is an ancillary subunit of the SecYEG translocon in Escherichia coli. J Biol Chem 289, 19089-19097 (2014).

20. B. Jauß et al., Non-competitive binding of PpiD and YidC to the SecYEG translocon expands the global view on the SecYEG interactome in E. coli. J Biol Chem, (2019).

21. N. A. Petriman et al., The interaction network of the YidC insertase with the SecYEG translocon, SRP and the SRP receptor FtsY. Sci Rep 8, 578 (2018).

22. R. J. Schulze et al., Membrane protein insertion and proton-motive-force-dependent secretion through the bacterial holo-translocon SecYEG-SecDF-YajC-YidC. Proc Natl Acad Sci U S A 111, 4844-4849 (2014).

23. J. Oswald, R. Njenga, A. Natriashvili, P. Sarmah, H. G. Koch, The Dynamic SecYEG Translocon. Front Mol Biosci 8, 664241 (2021).

24. J. Komar et al., Membrane protein insertion and assembly by the bacterial holotranslocon Sec YEG-SecDF-YajC-YidC. Biochem J 473, 3341-3354 (2016).

25. F. Duong, Capturing the bacterial holo-complex. Proc Natl Acad Sci U S A 111, 47394740 (2014).

26. I. Collinson, The Dynamic ATP-Driven Mechanism of Bacterial Protein Translocation and the Critical Role of Phospholipids. Front Microbiol 10, 1217 (2019).

27. M. Botte et al., A central cavity within the holo-translocon suggests a mechanism for membrane protein insertion. Sci Rep 6, 38399 (2016).

28. J. Young, F. Duong, Investigating the stability of the SecA-SecYEG complex during protein translocation across the bacterial membrane. J Biol Chem 294, 3577-3587 (2019).

29. X. X. Zhang et al., Nanodiscs and SILAC-based mass spectrometry to identify a membrane protein interactome. J Proteome Res 11, 1454-1459 (2012).

30. F. Duong, W. Wickner, The SecDFyajC domain of preprotein translocase controls preprotein movement by regulating SecA membrane cycling. EMBO J 16, 4871-4879 (1997).

31. H. E. Klock, S. A. Lesley, The Polymerase Incomplete Primer Extension (PIPE) method applied to high-throughput cloning and site-directed mutagenesis. Methods Mol Biol 498, 91-103 (2009).

32. S. Tyanova, T. Temu, J. Cox, The MaxQuant computational platform for mass spectrometry-based shotgun proteomics. Nat Protoc 11, 2301-2319 (2016).

33. J. Cox, M. Mann, MaxQuant enables high peptide identification rates, individualized p.p.b.-range mass accuracies and proteome-wide protein quantification. Nat Biotechnol 26, 1367-1372 (2008).

34. M. L. Carlson et al., The Peptidisc, a simple method for stabilizing membrane proteins in detergent-free solution. Elife 7, (2018). 
35. K. Dalal, C. S. Chan, S. G. Sligar, F. Duong, Two copies of the SecY channel and acidic lipids are necessary to activate the SecA translocation ATPase. Proc Natl Acad Sci U S A 109, 4104-4109 (2012).

36. C. Bechara et al., A subset of annular lipids is linked to the flippase activity of an ABC transporter. Nat Chem 7, 255-262 (2015).

37. W. Mi et al., Structural basis of MsbA-mediated lipopolysaccharide transport. Nature 549, 233-237 (2017).

38. B. Jauss et al., Noncompetitive binding of PpiD and YidC to the SecYEG translocon expands the global view on the SecYEG interactome in. J Biol Chem 294, 1916719183 (2019).

39. V. I. Perivolidi et al., Proteomic Identification of the SLC25A46 Interactome in Transgenic Mice Expressing SLC25A46-FLAG. J Proteome Res, (2022).

40. X. Liu et al., An AP-MS- and BioID-compatible MAC-tag enables comprehensive mapping of protein interactions and subcellular localization. Nat Commun 9, 1188 (2018). 


\section{FIGURES}

Native Membranes

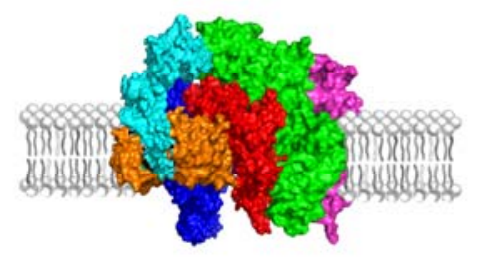

Detergent

Solubilization

Dissociation

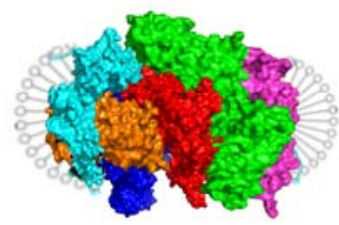

Peptidisc Stabilization

$+$
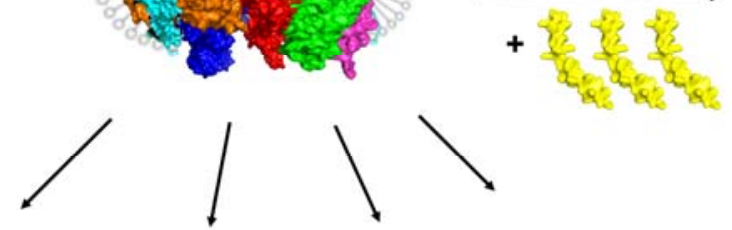

$\backslash \searrow$
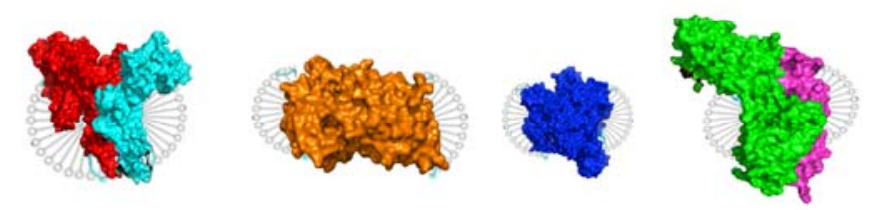

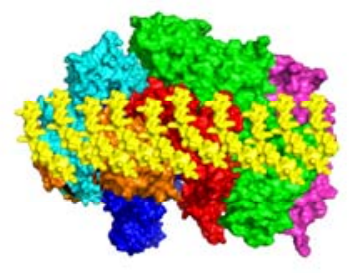

Figure 1: Stabilization of multi-subunit membrane protein complexes using the peptidisc. A multi-subunit membrane protein complex is initially extracted from the lipid bilayer with detergents. However, the complex is prone to dissociation into sub-complexes following prolonged detergent exposure. Peptidisc reconstitution enables stabilization of the assembly for downstream purification. 

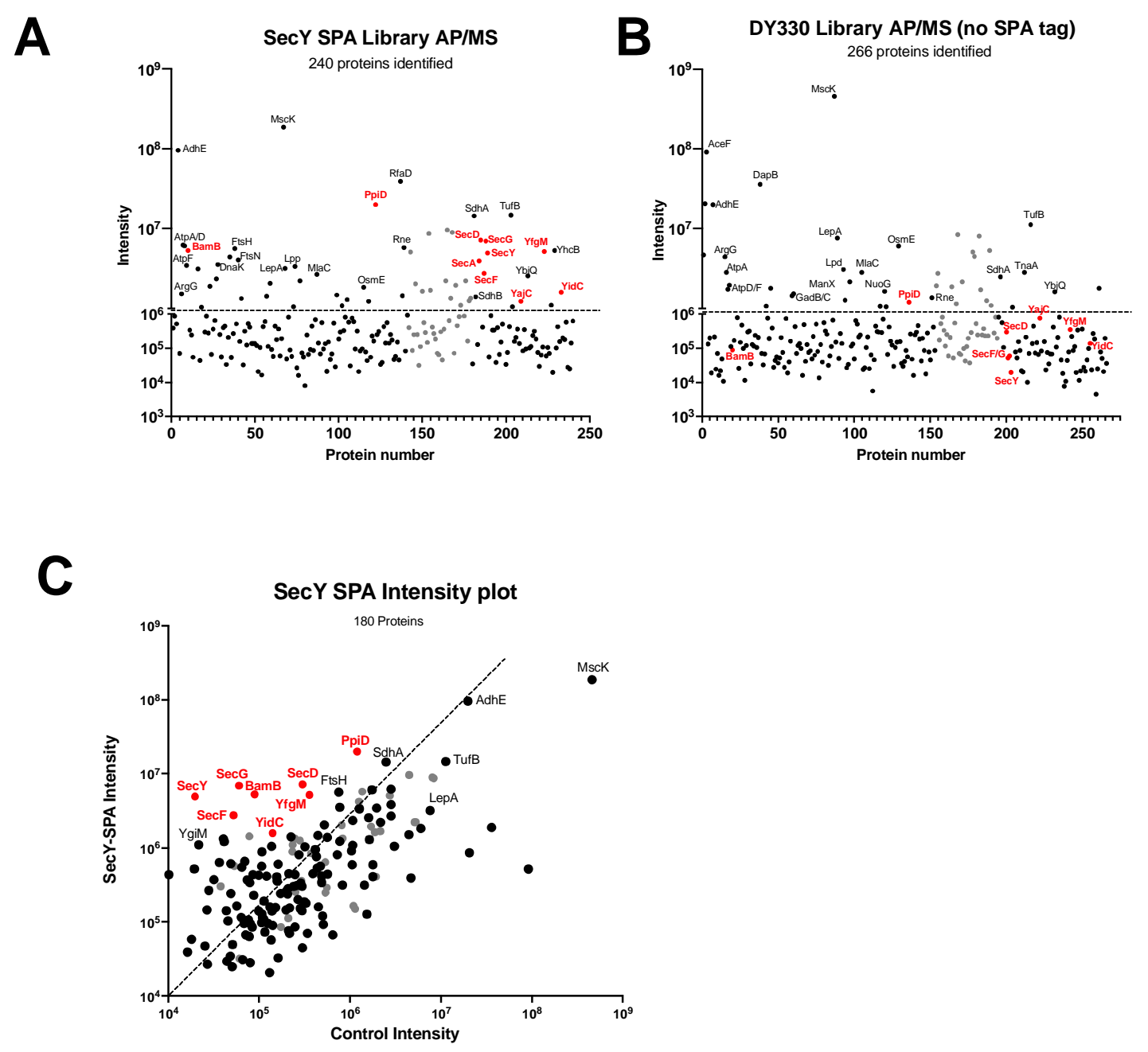

Figure 2: Capture and identification of SPA-tagged SecY using FLAG antibodies.

A. Peptidisc libraries prepared from an E. coli strain modified with a chromosomally SPAtagged SecY protein were purified over ANTI-FLAG M2 Agarose beads. LC-MS/MS identified eluted proteins. The protein list was ranked alphabetically to visualize the data, and protein intensities were plotted against protein rank in the list. Proteins of interest are highlighted in red; ribosomal subunits are in grey; all other identified proteins are shown in black. B. To identify non-specific protein contaminants, a peptidisc library prepared from the parental strain (no SPA tag) was processed in parallel. Data were analyzed and plotted as in (A). C. To give an idea of protein enrichment, the intensity of each protein present in the SecY sample was plotted against its intensity in the control sample. Colour coding was as described in (A). 
A

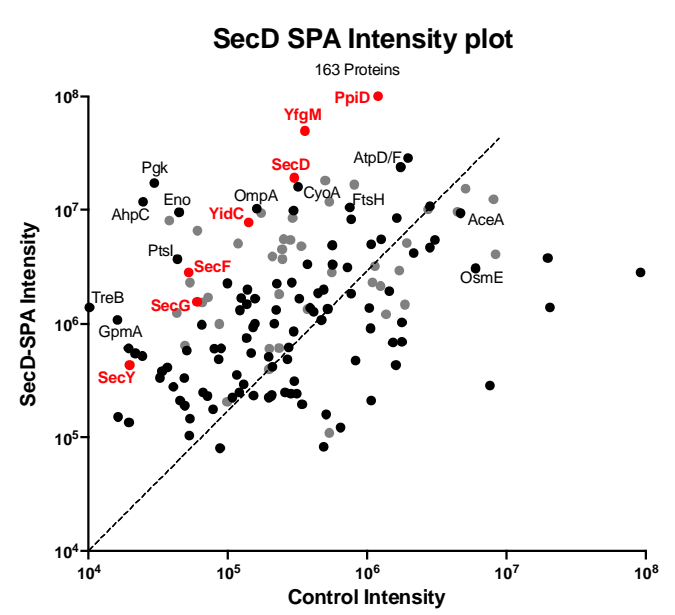

C

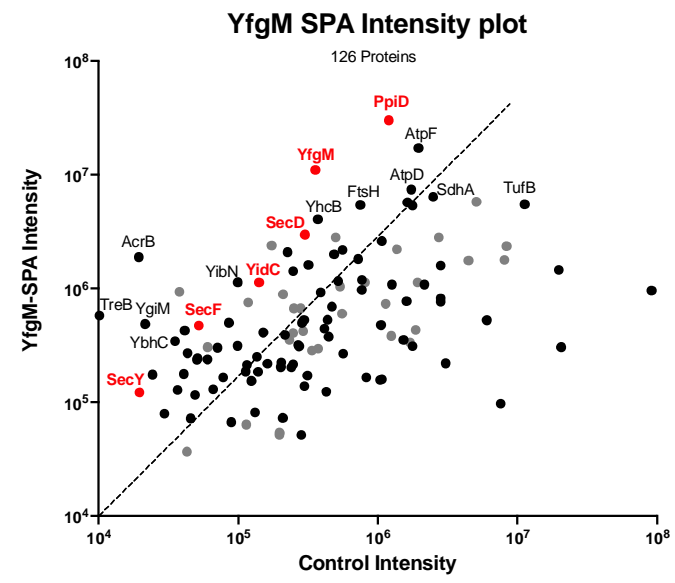

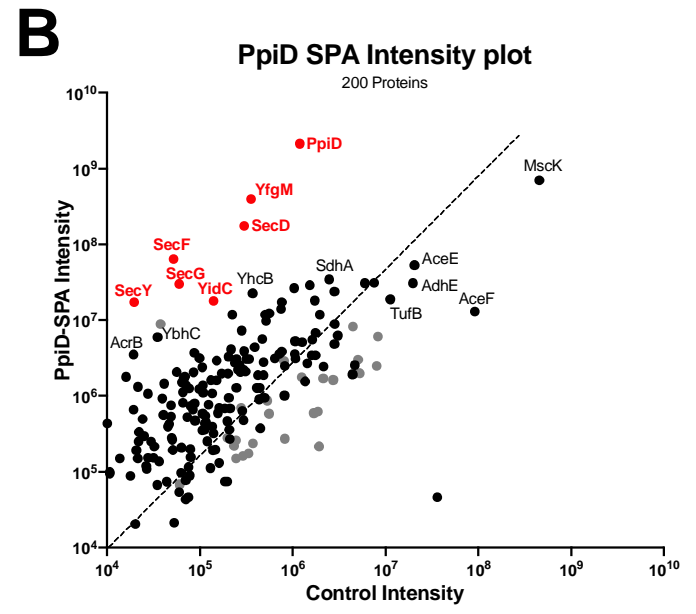

Figure 3: Capture and identification of Sec translocon ancillary subunits by FLAG AP/MS A. Capture and identification of SPA-tagged SecD using FLAG antibodies. Peptidisc libraries prepared from an E. coli strain modified with a chromosomally SPAtagged SecD protein were purified over ANTI-FLAG M2 Agarose beads. Data were analyzed and visualized as in Figure 4C. B. As in (A), but for SPA-tagged PpiD. C. As in (A) but for SPA-tagged YfgM. 

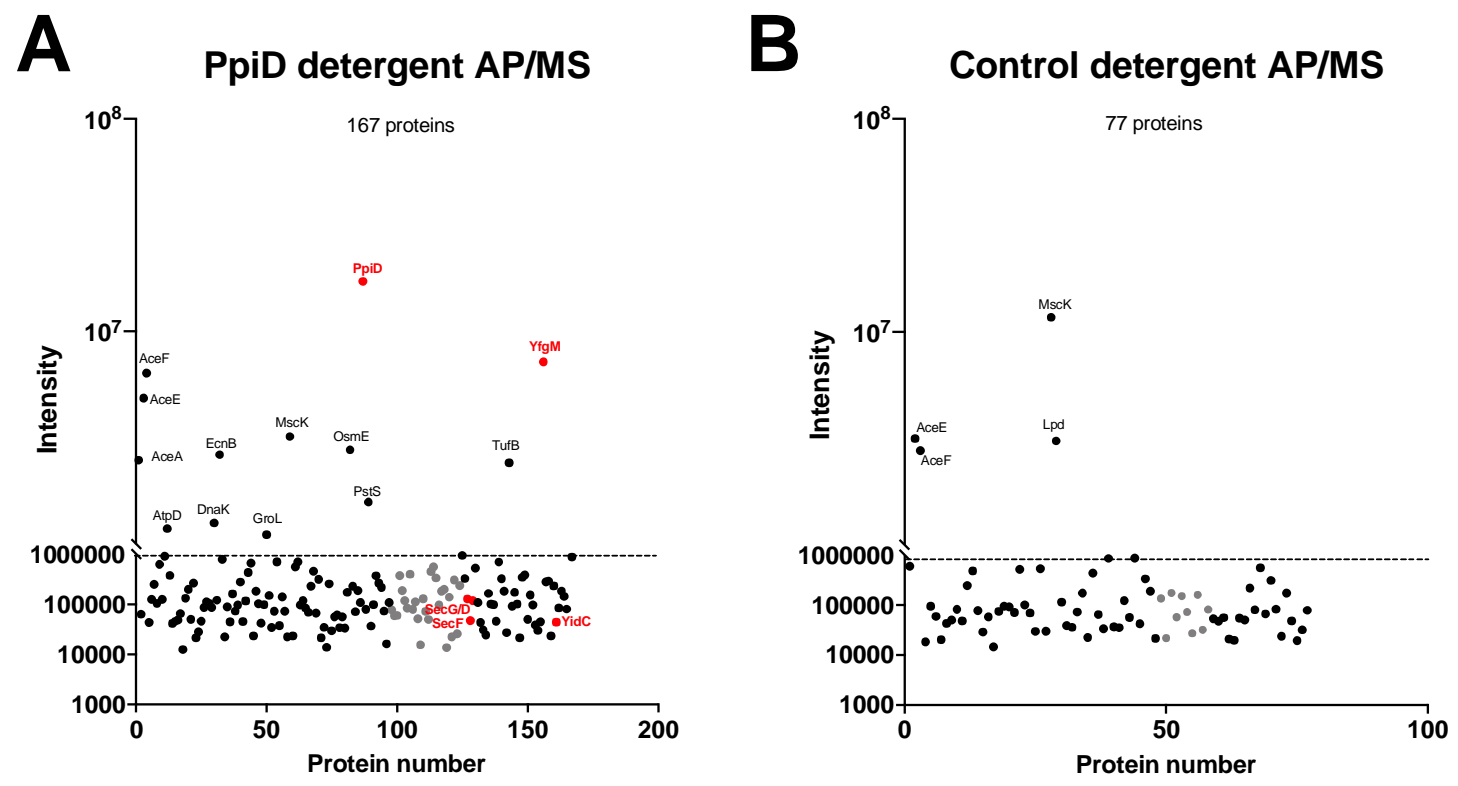

\section{C}

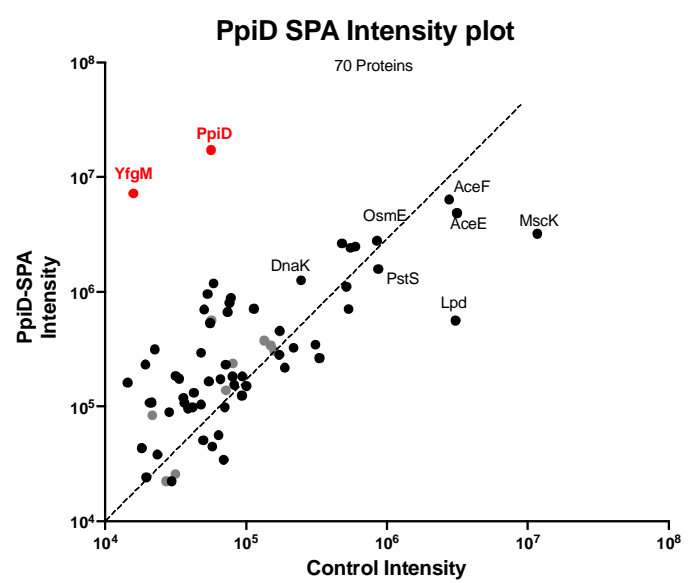

Figure 4: Capture and identification of SPA-tagged PpiD in DDM using FLAG antibodies. A. A detergent extract prepared from an E. coli strain modified with a chromosomally SPA-tagged PpiD protein was purified over ANTI-FLAG M2 Agarose beads. Data were analyzed and visualized as described in Figure 2A. B. To identify non-specific protein contaminants, a detergent extract prepared from the parental strain (no SPA tag) was processed in parallel. Data were analyzed and plotted as in (A). C. To give an idea of protein 
enrichment, the intensity of each protein present in the PpiD sample was plotted against its intensity in the control sample. Colour coding was as described in (A).
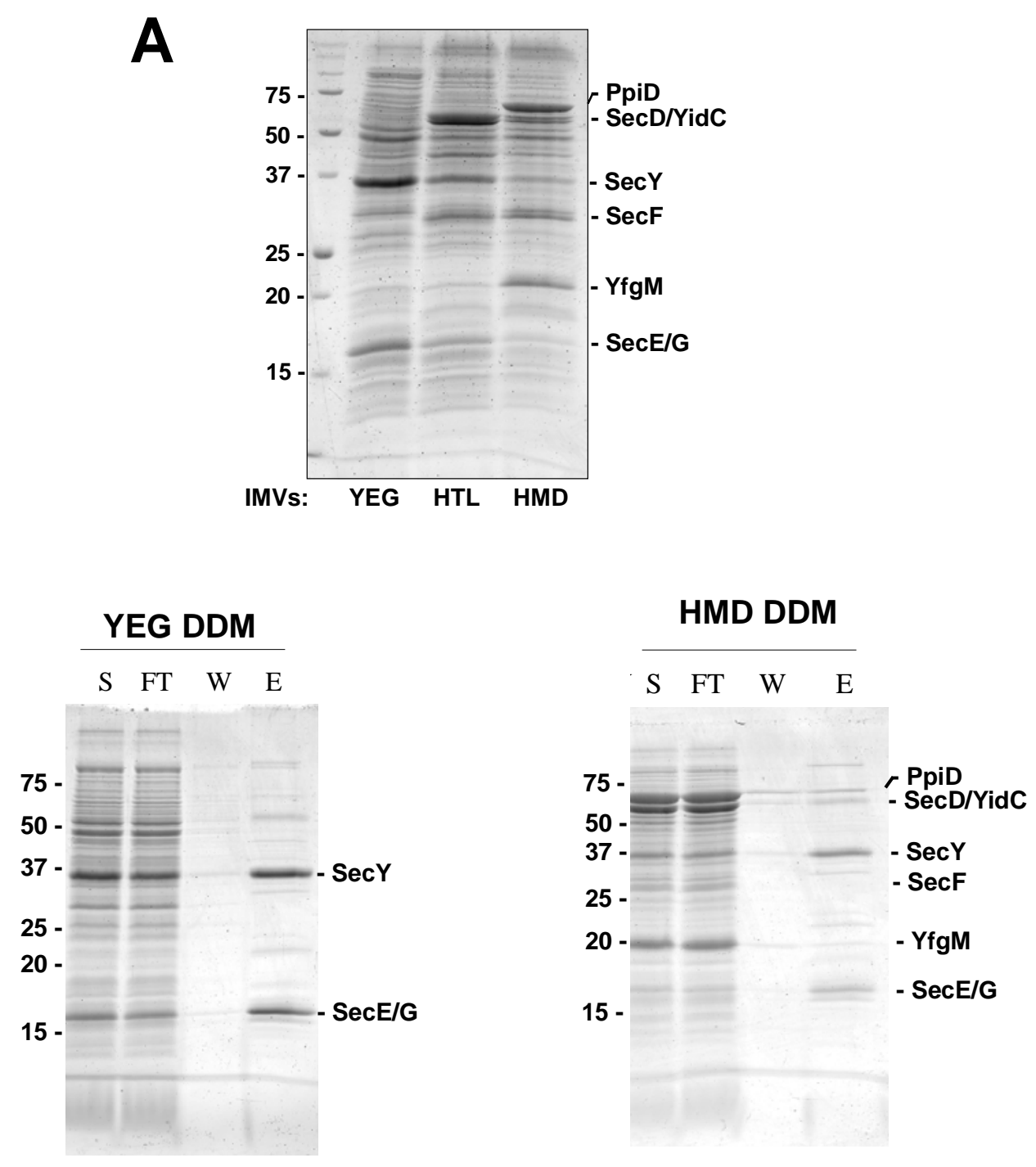

Figure 5: Detergent sensitivity of the "HMD" complex. A. SDS-PAGE analysis of $E$. coli inner membranes containing the over-expressed SecYEG, HTL, and HMD complexes.

Migration of each over-expressed protein subunit is indicated on the right side of the gel. $\mathbf{B}$. Membranes containing SecYEG and HMD from (A) were solubilized in DDM and purified over Ni-NTA affinity resin. Aliquots of each purification were analyzed by SDS-PAGE and Coomassie staining $(\mathrm{S}=$ untreated start material; $\mathrm{FT}=$ unbound flowthrough; $\mathrm{W}=$ wash; $\mathrm{E}=$ elution). 

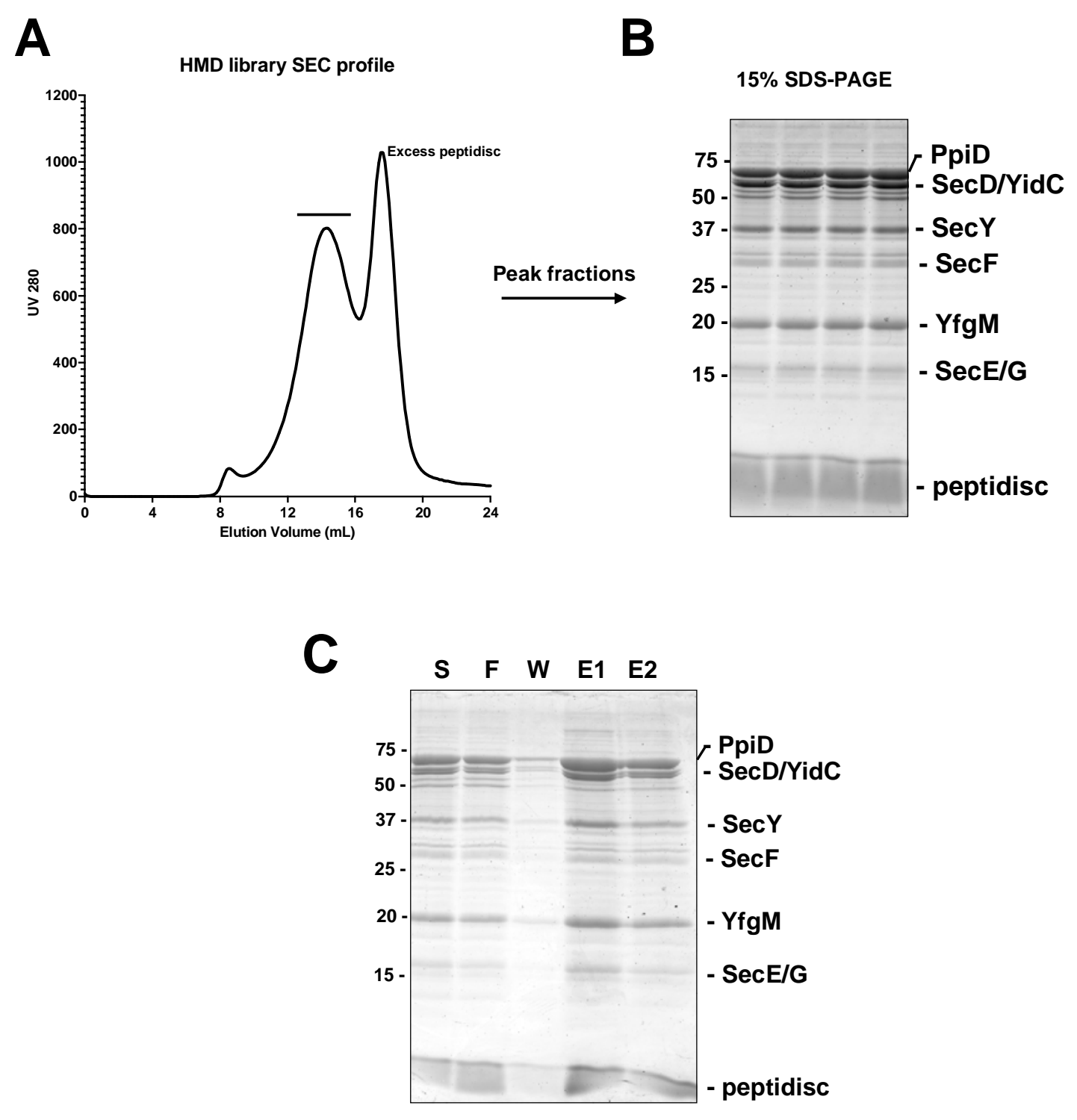

Figure 6: Purification of the "HMD" complex A. A peptidisc library containing the HMD complex was injected on a Superose $610 / 300$ column equilibrated in a detergent-free buffer. Protein abundance was monitored by UV $(280 \mathrm{~nm})$ and plotted as a function of elution volume $(\mathrm{mL})$. Large aggregates are expected to elute at $8 \mathrm{~mL}$ - the void volume of the column. B. Fractions under the central peak (indicated by a black bar in (A) were analyzed by SDS-PAGE and Coomassie staining. Migration of over-expressed protein subunits is indicated on the right-hand side of the gel. C. Peak fractions from (B) were pooled, and the HMD complex was isolated by affinity pulldown. 
bioRxiv preprint doi: https://doi.org/10.1101/2022.03.03.482899; this version posted March 3, 2022. The copyright holder for this preprint (which was not certified by peer review) is the author/funder. All rights reserved. No reuse allowed without permission. 